\title{
The composition of Europa's near-surface atmosphere
}

\author{
Mau C. Wong ${ }^{1}$, Tim Cassidy ${ }^{2}$, and Robert E. Johnson ${ }^{2}$ \\ ${ }^{1}$ Jet Propulsion Laboratory, California Institute of Technology, Pasadena CA, USA \\ email: mau.c.wong@jpl.nasa.gov \\ ${ }^{2}$ University of Virginia, Charlottesville VA, USA
}

\begin{abstract}
The presence of an undersurface ocean renders Europa as one of the few planetary bodies in our Solar System that has been conjectured to have possibly harbored life. Some of the organic and inorganic species present in the ocean underneath are expected to transport upwards through the relatively thin ice crust and manifest themselves as impurities of the water ice surface. For this reason, together with its unique dynamic atmosphere and geological features, Europa has attracted strong scientific interests in past decades.

Europa is imbedded inside the Jovian magnetosphere, and, therefore, is constantly subjected to the immerse surrounding radiations, similar to the other three Galilean satellites. The magnetosphere-atmosphere-surface interactions form a complex system that provides a multitude of interesting geophysical phenomenon that is unique in the Solar System. The atmosphere of Europa is thought to have created by, mostly, charged particles sputtering of surface materials. Consequently, the study of Europa's atmosphere can be used as a tool to infer the surface composition. In this paper, we will discuss our recent model studies of Europa's near-surface atmosphere. In particular, the abundances and distributions of the dominant $\mathrm{O}_{2}$ and $\mathrm{H}_{2} \mathrm{O}$ species, and of other organic and inorganic minor species will be addressed.
\end{abstract}

\section{Discussion}

Mumma: I did my thesis work on electron scattering from simple molecular gases, including $\mathrm{O}_{2}, \mathrm{CO}_{2}$, and so forth, in the vacuum ultraviolet. The line that Melissa McGrath reported at $1356 \AA$ is the quintet S triple $\mathrm{S}$ transition in atomic oxygen. It doesn't have to be produced from $\mathrm{O}_{2}$, because $\mathrm{H}_{2} \mathrm{O}$ can produce atomic oxygen quite easily through UV photolysis.

Wong: I think the way they conclude it is $\mathrm{O}_{2}$ is from the ratio between the $1356 \AA$ and $1304 \AA$ lines. They look at the ratio of these two lines and conclude it's most likely from the $\mathrm{O}_{2}$ dissociation. 


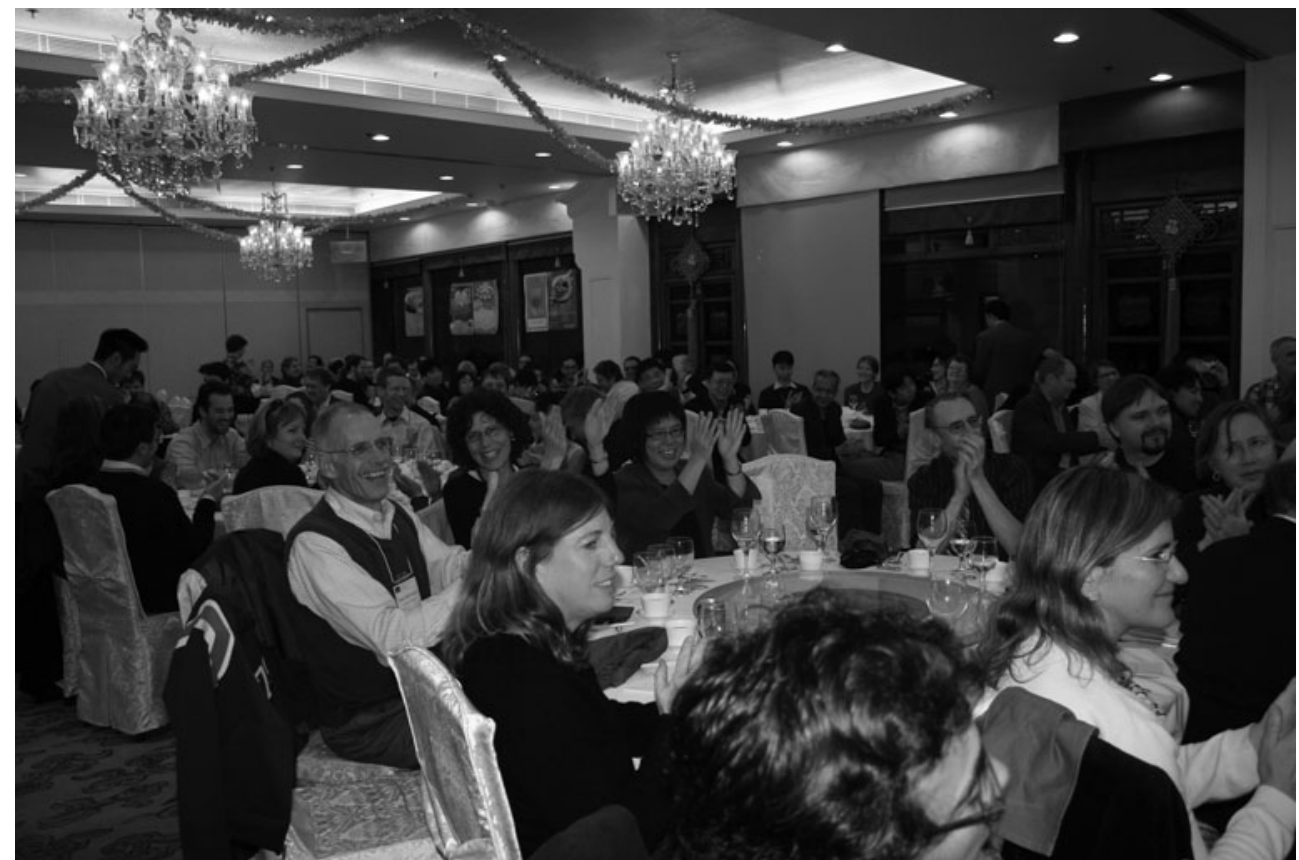

Participants applauding the singing of the prisoner of war song by Cliff Matthews during the banquet. 